\title{
Fixed point theorems for a class of mixed monotone operators with convexity
}

\section{Chengbo Zhai*}

\section{"Correspondence:}

cbzhai@sxu.edu.cn;

cbzhai215@sohu.com

School of Mathematical Sciences,

Shanxi University, Taiyuan, Shanxi

030006, P.R. China

\section{笔 Springer}

\begin{abstract}
In this paper, we use partial order theory to study a class of mixed monotone operators with convexity, and we get the existence and uniqueness of fixed points without assuming the operator to be compact or continuous. Our results compliment the theory of mixed monotone operators in ordered Banach spaces.

MSC: $47 \mathrm{H} 10 ; 47 \mathrm{H} 07$

Keywords: fixed point; mixed monotone operator; normal cone; convexity; $\alpha$-convex operator
\end{abstract}

\section{Introduction and preliminaries}

It is well known that mixed monotone operators were introduced by Guo and Lakshmikantham [1] in 1987. Thereafter many authors have investigated these kinds of operators in Banach spaces and obtained a lot of interesting and important results (see [1-18] and the references therein). Their study has not only important theoretical meaning but also wide applications in engineering, nuclear physics, biological chemistry technology, etc. That is, they are used extensively in nonlinear differential and integral equations. For a small sample of such work, we refer the reader to works [13, 15-21]. However, the mixed monotone operators considered in most of these papers are concave; see [2-4, 7-11, 15, $17,18]$, for instance. To our knowledge, the fixed point results on mixed monotone operators with convexity are still very few. So it is worthwhile to investigate this operator. The purpose of this paper is to establish the existence and uniqueness of fixed points for a class of mixed monotone operators with convexity. We will use partial order theory to study the mixed monotone operator with convexity and get the existence and uniqueness of fixed points without assuming the operator to be compact or continuous. Our results compliment the theory of mixed monotone operators in ordered Banach spaces.

For the discussion of the following section, we state here some definitions and notations. For convenience of readers, we suggest that one refers to $[1,2,17,21-23]$ for details.

Suppose that $(E,\|\cdot\|)$ is a real Banach space which is partially ordered by a cone $P \subset E$, i.e., $x \leq y$ if and only if $y-x \in P$. If $x \leq y$ and $x \neq y$, then we denote $x<y$ or $y>x$. By $\theta$ we denote the zero element of $E$. Recall that a non-empty closed convex set $P \subset E$ is a cone if it satisfies (i) $x \in P, \lambda \geq 0 \Rightarrow \lambda x \in P$; (ii) $x \in P,-x \in P \Rightarrow x=\theta$.

Further, $P$ is called normal if there exists a constant $N>0$ such that, for all $x, y \in E, \theta \leq$ $x \leq y$ implies $\|x\| \leq N\|y\|$; in this case $N$ is called the normality constant of $P$. If $x_{1}, x_{2} \in E$, the set $\left[x_{1}, x_{2}\right]=\left\{x \in E \mid x_{1} \leq x \leq x_{2}\right\}$ is called the order interval between $x_{1}$ and $x_{2}$. We say that an operator $A: E \rightarrow E$ is increasing if $x \leq y$ implies $A x \leq A y$.

(c) 2013 Zhai; licensee Springer. This is an Open Access article distributed under the terms of the Creative Commons Attribution License (http://creativecommons.org/licenses/by/2.0), which permits unrestricted use, distribution, and reproduction in any medium, provided the original work is properly cited. 
Definition 1.1 (See $[1,2]) A: P \times P \rightarrow P$ is said to be a mixed monotone operator if $A(x, y)$ is increasing in $x$ and decreasing in $y$, i.e., $u_{i}, v_{i}(i=1,2) \in P, u_{1} \leq u_{2}, v_{1} \geq v_{2}$ imply $A\left(u_{1}, v_{1}\right) \leq A\left(u_{2}, v_{2}\right)$. The element $x \in P$ is called a fixed point of $A$ if $A(x, x)=x$.

\section{Main results}

In this section we consider the existence and uniqueness of fixed points for a class of mixed monotone operators in ordered Banach spaces.

Theorem 2.1 Let E be a real Banach space and let $P$ be a normal cone in $E . A: P \times P \rightarrow P$ is a mixed monotone operator which satisfies the following:

$\left(\mathrm{H}_{1}\right)$ for $t \in(0,1), x, y \in P$, there exists $\alpha(t, x, y) \in(1,+\infty)$ such that

$$
A(t x, y) \leq t^{\alpha(t, x, y)} A(x, y)
$$

$\left(\mathrm{H}_{2}\right)$ there exist $u_{0}, v_{0} \in P, r \in(0,1)$ such that

$$
u_{0} \leq r v_{0}, \quad A\left(u_{0}, v_{0}\right) \geq u_{0}, \quad A\left(v_{0}, u_{0}\right) \leq v_{0} .
$$

Then $A$ has a unique fixed point $u^{*}$ in $\left[u_{0}, r v_{0}\right]$. Moreover, constructing successively the sequences

$$
x_{n}=A\left(x_{n-1}, y_{n-1}\right), \quad y_{n}=A\left(y_{n-1}, x_{n-1}\right), \quad n=1,2, \ldots,
$$

for any initial values $x_{0}, y_{0} \in\left[u_{0}, r v_{0}\right]$, we have $\left\|x_{n}-u^{*}\right\| \rightarrow 0,\left\|y_{n}-u^{*}\right\| \rightarrow 0$ as $n \rightarrow \infty$.

Remark 2.1 (i) The operator $A$ which satisfies $\left(\mathrm{H}_{1}\right)$ can be called a mixed monotone operator with convexity; (ii) from $\left(\mathrm{H}_{1}\right)$, we can get $A(\theta, \theta)=\theta$. In fact, for $t_{0} \in(0,1)$, $A(\theta, \theta)=A\left(t_{0} \theta, \theta\right) \leq t_{0}^{\alpha\left(t_{0}, \theta, \theta\right)} A(\theta, \theta)$, and thus $\left[1-t_{0}^{\alpha\left(t_{0}, \theta, \theta\right)}\right] A(\theta, \theta) \leq \theta$. It follows from $A(\theta, \theta) \geq \theta$ that $A(\theta, \theta)=\theta$; (iii) from (2.1), we can get

$$
A\left(\frac{1}{t} x, y\right) \geq \frac{1}{t^{\alpha\left(t, \frac{1}{t} x, y\right)}} A(x, y), \quad x, y \in P, t \in(0,1) .
$$

Proof of Theorem 2.1 Let $w_{0}=r v_{0}, \varepsilon=r^{\alpha\left(r, v_{0}, u_{0}\right)-1}$. Then $w_{0} \geq u_{0}, \varepsilon \in(0,1)$, and

$$
\begin{aligned}
A\left(w_{0}, u_{0}\right) & =A\left(r v_{0}, u_{0}\right) \leq r^{\alpha\left(r, v_{0}, u_{0}\right)} A\left(v_{0}, u_{0}\right) \leq r^{\alpha\left(r, v_{0}, u_{0}\right)} v_{0}=r^{\alpha\left(r, v_{0}, u_{0}\right)-1} \cdot r v_{0} \\
& =\varepsilon w_{0} \leq w_{0}, \\
A\left(u_{0}, w_{0}\right) & =A\left(u_{0}, r v_{0}\right) \geq A\left(u_{0}, v_{0}\right) \geq u_{0} .
\end{aligned}
$$

Construct successively the sequences

$$
\begin{aligned}
& u_{n}=A\left(u_{n-1}, w_{n-1}\right), \quad w_{n}=A\left(w_{n-1}, u_{n-1}\right), \\
& w_{n}^{\prime}=\frac{1}{\varepsilon} A\left(w_{n-1}^{\prime}, u_{n-1}\right), \quad w_{0}^{\prime}=w_{0}, \quad n=1,2, \ldots
\end{aligned}
$$


From (2.4), (2.5) and the mixed monotonicity of $A$, we have

$$
u_{0} \leq u_{1} \leq u_{2} \leq \cdots \leq u_{n} \leq \cdots \leq w_{n} \leq \cdots \leq w_{1} \leq w_{0}
$$

Next we prove that

$$
u_{0} \leq w_{n}^{\prime} \leq w_{0}, \quad n=1,2, \ldots
$$

From (2.4),

$$
\begin{aligned}
& w_{1}^{\prime}=\frac{1}{\varepsilon} A\left(w_{0}, u_{0}\right) \leq \frac{1}{\varepsilon} \varepsilon w_{0}=w_{0}, \\
& w_{1}^{\prime}=\frac{1}{\varepsilon} A\left(w_{0}, u_{0}\right) \geq \frac{1}{\varepsilon} A\left(u_{0}, w_{0}\right) \geq \frac{1}{\varepsilon} u_{0} \geq u_{0}, \\
& w_{2}^{\prime}=\frac{1}{\varepsilon} A\left(w_{1}^{\prime}, u_{1}\right) \leq \frac{1}{\varepsilon} A\left(w_{0}, u_{0}\right) \leq \frac{1}{\varepsilon} \varepsilon w_{0}=w_{0}, \\
& w_{2}^{\prime}=\frac{1}{\varepsilon} A\left(w_{1}^{\prime}, u_{1}\right) \geq \frac{1}{\varepsilon} A\left(u_{0}, v_{0}\right) \geq \frac{1}{\varepsilon} u_{0} \geq u_{0} .
\end{aligned}
$$

Suppose that when $n=k$, we have $u_{0} \leq w_{k}^{\prime} \leq w_{0}$. Then, when $n=k+1$, we obtain

$$
\begin{aligned}
& w_{k+1}^{\prime}=\frac{1}{\varepsilon} A\left(w_{k}^{\prime}, u_{k}\right) \leq \frac{1}{\varepsilon} A\left(w_{0}, u_{0}\right) \leq \frac{1}{\varepsilon} \varepsilon w_{0}=w_{0}, \\
& w_{k+1}^{\prime}=\frac{1}{\varepsilon} A\left(w_{k}^{\prime}, u_{k}\right) \geq \frac{1}{\varepsilon} A\left(u_{0}, v_{0}\right) \geq \frac{1}{\varepsilon} u_{0} \geq u_{0} .
\end{aligned}
$$

By the induction method, we know that (2.7) holds. On the other hand, from (2.1),

$$
\begin{aligned}
& w_{1}=A\left(w_{0}, u_{0}\right)=\varepsilon \frac{1}{\varepsilon} A\left(w_{0}, u_{0}\right)=\varepsilon w_{1}^{\prime}, \\
& w_{2}=A\left(w_{1}, u_{1}\right)=A\left(\varepsilon w_{1}^{\prime}, u_{1}\right) \leq \varepsilon^{\alpha\left(\varepsilon, w_{1}^{\prime}, u_{1}\right)} A\left(w_{1}^{\prime}, u_{1}\right)=\varepsilon^{\alpha\left(\varepsilon, w_{1}^{\prime}, u_{1}\right)+1} \frac{1}{\varepsilon} A\left(w_{1}^{\prime}, u_{1}\right) \leq \varepsilon^{2} w_{2}^{\prime} .
\end{aligned}
$$

Suppose that when $n=k$, we have $w_{k} \leq \varepsilon^{k} w_{k}^{\prime}$. Then, when $n=k+1$, we obtain

$$
\begin{aligned}
w_{k+1} & =A\left(w_{k}, u_{k}\right) \leq A\left(\varepsilon^{k} w_{k}^{\prime}, u_{k}\right) \leq\left(\varepsilon^{k}\right)^{\alpha\left(\varepsilon^{k}, w_{k}^{\prime}, u_{k}\right)} A\left(w_{k}^{\prime}, u_{k}\right) \\
& =\varepsilon^{k \alpha\left(\varepsilon^{k}, w_{k}^{\prime}, u_{k}\right)+1} \frac{1}{\varepsilon} A\left(w_{k}^{\prime}, u_{k}\right) \leq \varepsilon^{k+1} w_{k+1}^{\prime} .
\end{aligned}
$$

By the induction method, we have

$$
w_{n} \leq \varepsilon^{n} w_{n}^{\prime}, \quad n=1,2, \ldots
$$

By (2.6)-(2.8), we get

$$
\begin{aligned}
& \theta \leq w_{n}-u_{n} \leq \varepsilon^{n} w_{n}^{\prime}-u_{n} \leq \varepsilon^{n} w_{n}^{\prime}-\varepsilon^{n} u_{n}=\varepsilon^{n}\left(w_{n}^{\prime}-u_{n}\right) \leq \varepsilon^{n}\left(w_{0}-u_{0}\right), \\
& \theta \leq u_{n+p}-u_{n} \leq w_{n}-u_{n}, \quad \theta \leq w_{n}-w_{n+p} \leq w_{n}-u_{n} .
\end{aligned}
$$


Since $P$ is normal, we have

$$
\left\|w_{n}-u_{n}\right\| \leq N \varepsilon^{n}\left\|w_{0}-u_{0}\right\| \rightarrow 0 \quad(\text { as } n \rightarrow \infty)
$$

Further,

$$
\begin{aligned}
& \left\|u_{n+p}-u_{n}\right\| \leq N\left\|w_{n}-u_{n}\right\| \rightarrow 0 \quad(\text { as } n \rightarrow \infty), \\
& \left\|w_{n}-w_{n+p}\right\| \leq N\left\|w_{n}-u_{n}\right\| \rightarrow 0 \quad(\text { as } n \rightarrow \infty) .
\end{aligned}
$$

Here $N$ is the normality constant.

So, we can claim that $\left\{u_{n}\right\}$ and $\left\{w_{n}\right\}$ are Cauchy sequences. Because $E$ is complete, there exist $u^{*}, w^{*} \in P$ such that

$$
u_{n} \rightarrow u^{*}, \quad w_{n} \rightarrow w^{*}, \quad \text { as } n \rightarrow \infty .
$$

By (2.6), we know that $u_{0} \leq u_{n} \leq u^{*} \leq w^{*} \leq w_{n} \leq w_{0}$, and then

$$
\theta \leq w^{*}-u^{*} \leq w_{n}-u_{n} \leq \varepsilon^{n}\left(w_{0}-u_{0}\right) \text {. }
$$

Further, $\left\|w^{*}-u^{*}\right\| \leq N \varepsilon^{n}\left\|w_{0}-u_{0}\right\| \rightarrow 0$ (as $\left.n \rightarrow \infty\right)$, and thus $w^{*}=u^{*}$. Then we obtain

$$
u_{n+1}=A\left(u_{n}, w_{n}\right) \leq A\left(u^{*}, u^{*}\right) \leq A\left(w_{n}, u_{n}\right)=w_{n+1} .
$$

Let $n \rightarrow \infty$, then we get $A\left(u^{*}, u^{*}\right)=u^{*}$. That is, $u^{*}$ is a fixed point of $A$ in $\left[u_{0}, w_{0}\right]=\left[u_{0}, r v_{0}\right]$. In the following, we prove that $u^{*}$ is the unique fixed point of $A$ in $\left[u_{0}, w_{0}\right]$. Suppose that there is $x^{*} \in\left[u_{0}, w_{0}\right]$ such that $A\left(x^{*}, x^{*}\right)=x^{*}$. Then we have $u_{0} \leq x^{*} \leq w_{0}$. By the induction method and the mixed monotonicity of $A$, we have

$$
u_{n+1}=A\left(u_{n}, w_{n}\right) \leq x^{*}=A\left(x^{*}, x^{*}\right) \leq A\left(w_{n}, u_{n}\right)=w_{n+1}, \quad n=0,1,2, \ldots
$$

Then from the normality of $P$, we have $x^{*}=u^{*}$.

Moreover, constructing successively the sequences

$$
x_{n}=A\left(x_{n-1}, y_{n-1}\right), \quad y_{n}=A\left(y_{n-1}, x_{n-1}\right), \quad n=1,2, \ldots,
$$

for any initial values $x_{0}, y_{0} \in\left[u_{0}, w_{0}\right]$, we have $u_{n} \leq x_{n}, y_{n} \leq w_{n}, n=1,2, \ldots$ Letting $n \rightarrow \infty$ yields $x_{n} \rightarrow u^{*}, y_{n} \rightarrow u^{*}$ as $n \rightarrow \infty$.

Remark 2.2 Let $\alpha(t, x, y)$ be a constant $\alpha \in(1,+\infty)$, then Theorem 2.1 also holds.

Corollary 2.2 Let E be a real Banach space and let $P$ be a normal cone in $E . A: P \times P \rightarrow P$ is a mixed monotone operator which satisfies $\left(\mathrm{H}_{2}\right)$ and, for $t \in(0,1), x, y \in P$, there exists a constant $\alpha \in(1,+\infty)$ such that $A(t x, y) \leq t^{\alpha} A(x, y)$. Then $A$ has a unique fixed point $u^{*}$ in $\left[u_{0}, r v_{0}\right]$. Moreover, constructing successively the sequences

$$
x_{n}=A\left(x_{n-1}, y_{n-1}\right), \quad y_{n}=A\left(y_{n-1}, x_{n-1}\right), \quad n=1,2, \ldots,
$$

for any initial values $x_{0}, y_{0} \in\left[u_{0}, r v_{0}\right]$, we have $\left\|x_{n}-u^{*}\right\| \rightarrow 0,\left\|y_{n}-u^{*}\right\| \rightarrow 0$ as $n \rightarrow \infty$. 
From the proof of Theorem 2.1, we can easily obtain the following conclusion.

Corollary 2.3 Let E be a real Banach space and let P be a normal cone in $E . A: P \rightarrow P$ is an increasing operator which satisfies the following:

$\left(\mathrm{H}_{3}\right)$ for $t \in(0,1), x \in P$, there exists a constant $\alpha \in(1,+\infty)$ such that $A(t x) \leq t^{\alpha} A x$;

$\left(\mathrm{H}_{4}\right)$ there exist $u_{0}, v_{0} \in P, r \in(0,1)$ such that $u_{0} \leq r v_{0}, A u_{0} \geq u_{0}, A v_{0} \leq v_{0}$.

Then $A$ has a unique fixed point $u^{*}$ in $\left[u_{0}, r v_{0}\right]$. Moreover, constructing successively the sequence

$$
x_{n}=A x_{n-1}, \quad n=1,2, \ldots,
$$

for any initial value $x_{0} \in\left[u_{0}, r v_{0}\right]$, we have $\left\|x_{n}-u^{*}\right\| \rightarrow 0$ as $n \rightarrow \infty$.

Remark 2.3 The operator $A$ which satisfies $\left(\mathrm{H}_{3}\right)$ is called $\alpha$-convex. In 1977, A.J.B. Potter introduced the definition of an $\alpha$-convex operator and showed that for $\alpha \geq 0$, decreasing $(-\alpha)$-convex mappings have contraction ratios less than or equal to $\alpha$ and gave the existence of solutions to the nonlinear eigenvalue problem $A x=\lambda x$. The method is based upon Hilbert's projective metric. In [22] Guo studied the existence and uniqueness of fixed points for $(-\alpha)$-convex operators. In [23], we obtained the existence and uniqueness of positive fixed points for $\alpha$-convex operators by means of the properties of cone, concave operators and the monotonicity of set-valued maps. Here, the result on $\alpha$-convex operators is new and the method is also new and different from previous ones.

Remark 2.4 If $A: P \rightarrow P$ is an $\alpha$-convex operator, then $A$ is not a decreasing and constant operator. In fact, suppose that $A$ is decreasing, then we have $A x \leq A(t x) \leq t^{\alpha} A x, x \in P$, $t \in(0,1)$. Hence, $t^{\alpha} \geq 1$, this is a contradiction. Suppose that $A$ is a constant operator, that is, $A x=u_{0} \in P$. Then $u_{0}=A(t x) \leq t^{\alpha} A x=t^{\alpha} u_{0}$, and thus $t^{\alpha} \geq 1$, this is also a contradiction.

Theorem 2.4 Let E be a real Banach space and let P be a normal cone in $E . A: P \times P \rightarrow P$ is a mixed monotone operator which satisfies (2.1) and

$\left(\mathrm{H}_{5}\right)$ there exist $u_{0}, v_{0} \in P, R \in(1,+\infty)$ such that

$$
A\left(u_{0}, v_{0}\right) \geq u_{0}, \quad A\left(v_{0}, u_{0}\right) \leq v_{0}, \quad v_{0} \geq R u_{0} .
$$

Then the operator equation $A(u, u)=b u$ has a unique solution $u^{*}$ in $\left[R u_{0}, v_{0}\right]$, where $b=$ $R^{\alpha\left(\frac{1}{R}, R u_{0}, v_{0}\right)-1}$. Moreover, constructing successively the sequences

$$
x_{n}=b^{-1} A\left(x_{n-1}, y_{n-1}\right), \quad y_{n}=b^{-1} A\left(y_{n-1}, x_{n-1}\right), \quad n=1,2, \ldots,
$$

for any initial values $x_{0}, y_{0} \in\left[R u_{0}, v_{0}\right]$, we have $\left\|x_{n}-u^{*}\right\| \rightarrow 0,\left\|y_{n}-u^{*}\right\| \rightarrow 0$ as $n \rightarrow \infty$. 
Proof Let $w_{0}=R u_{0}$. Then $w_{0} \leq v_{0}$. Note that $b>1$ and from (2.3),

$$
\begin{aligned}
A\left(w_{0}, v_{0}\right) & =A\left(R u_{0}, v_{0}\right) \geq \frac{1}{\left(\frac{1}{R}\right)^{\alpha\left(\frac{1}{R}, R u_{0}, v_{0}\right)}} A\left(u_{0}, v_{0}\right) \\
& \geq R^{\alpha\left(\frac{1}{R}, R u_{0}, v_{0}\right)} u_{0}=R^{\alpha\left(\frac{1}{R}, R u_{0}, v_{0}\right)-1} \cdot R u_{0}=b w_{0} \geq w_{0}, \\
A\left(v_{0}, w_{0}\right) & =A\left(v_{0}, R u_{0}\right) \leq A\left(v_{0}, u_{0}\right) \leq v_{0} .
\end{aligned}
$$

Set $B(x, y)=b^{-1} A(x, y), x, y \in P$. Then from the above inequalities, we have

$$
\begin{aligned}
& B\left(w_{0}, v_{0}\right)=b^{-1} A\left(w_{0}, v_{0}\right) \geq b^{-1} b w_{0}=w_{0}, \\
& B\left(v_{0}, w_{0}\right)=b^{-1} A\left(v_{0}, w_{0}\right) \leq b^{-1} v_{0} \leq v_{0} .
\end{aligned}
$$

Also, construct successively the sequences

$$
\begin{aligned}
& w_{n}=B\left(w_{n-1}, v_{n-1}\right), \quad v_{n}=B\left(v_{n-1}, w_{n-1}\right), \\
& v_{n}^{\prime}=b B\left(v_{n-1}^{\prime}, w_{n-1}\right), \quad v_{0}^{\prime}=v_{0}, \quad n=1,2, \ldots
\end{aligned}
$$

From (2.9) and the mixed monotonicity of $A$, we have

$$
w_{0} \leq w_{1} \leq w_{2} \leq \cdots \leq w_{n} \leq \cdots \leq v_{n} \leq \cdots \leq v_{1} \leq v_{0} .
$$

Similar to the proof of Theorem 2.1, we can prove that

$$
w_{0} \leq v_{n}^{\prime} \leq v_{0}, \quad v_{n} \leq\left(\frac{1}{b}\right)^{n} v_{n}^{\prime}, \quad n=1,2, \ldots
$$

Further, by using the same method with the proof of Theorem 2.1, we can get the following conclusions: (i) $B$ has a unique fixed point $u^{*}$ in $\left[w_{0}, v_{0}\right]$; (ii) for any initial values $x_{0}, y_{0} \in$ $\left[w_{0}, v_{0}\right]$, constructing successively the sequences

$$
x_{n}=B\left(x_{n-1}, y_{n-1}\right), \quad y_{n}=B\left(y_{n-1}, x_{n-1}\right), \quad n=1,2, \ldots
$$

we have $\left\|x_{n}-u^{*}\right\| \rightarrow 0,\left\|y_{n}-u^{*}\right\| \rightarrow 0$ as $n \rightarrow \infty$. Therefore, the operator equation $A(u, u)=b u$ has a unique solution $u^{*}$ in $\left[R u_{0}, v_{0}\right]$. Moreover, constructing successively the sequences

$$
x_{n}=b^{-1} A\left(x_{n-1}, y_{n-1}\right), \quad y_{n}=b^{-1} A\left(y_{n-1}, x_{n-1}\right), \quad n=1,2, \ldots,
$$

for any initial values $x_{0}, y_{0} \in\left[R u_{0}, v_{0}\right]$, we have $\left\|x_{n}-u^{*}\right\| \rightarrow 0,\left\|y_{n}-u^{*}\right\| \rightarrow 0$ as $n \rightarrow \infty$.

Corollary 2.5 Let $E$ be a real Banach space and let $P$ be a normal cone in $E . A: P \rightarrow P$ is an increasing operator which satisfies $\left(\mathrm{H}_{3}\right)$ and

$\left(\mathrm{H}_{6}\right)$ there exist $u_{0}, v_{0} \in P, R \in(1,+\infty)$ such that $A u_{0} \geq u_{0}, A v_{0} \leq v_{0}, v_{0} \geq R u_{0}$. 
Then the operator equation $A u=b u$ has a unique solution $u^{*}$ in $\left[R u_{0}, v_{0}\right]$, where $b=R^{\alpha-1}$. Moreover, constructing successively the sequence

$$
x_{n}=b^{-1} A x_{n-1}, \quad n=1,2, \ldots,
$$

for any initial value $x_{0} \in\left[R u_{0}, v_{0}\right]$, we have $\left\|x_{n}-u^{*}\right\| \rightarrow 0$ as $n \rightarrow \infty$.

\section{Competing interests}

The author declares that he has no competing interests.

\section{Acknowledgements}

The author was supported financially by the Youth Science Foundations of China (11201272) and Shanxi Province (2010021002-1).

\section{Received: 7 January 2013 Accepted: 17 April 2013 Published: 3 May 2013}

\section{References}

1. Guo, D, Lakskmikantham, V: Coupled fixed points of nonlinear operators with applications. Nonlinear Anal. 11(5), 623-632 (1987)

2. Guo, D: Fixed points of mixed monotone operators with application. Appl. Anal. 34, 215-224 (1988)

3. Chen, Y: Thompson's metric and mixed monotone operators. J. Math. Anal. Appl. 177, 31-37 (1993)

4. Zhang, Z: New fixed point theorems of mixed monotone operators and applications. J. Math. Anal. Appl. 204 307-319 (1996)

5. Zhang, S, Ma, Y: Coupled fixed points for mixed monotone condensing operators and an existence theorem of the solution for a class of functional equations arising in dynamic programming. J. Math. Anal. Appl. 160, 468-479 (1991)

6. Sun, Y: A fixed point theorem for mixed monotone operator with applications. J. Math. Anal. Appl. 156, 240-252 (1991)

7. Liang, Z, Zhang, L, Li, S: Fixed point theorems for a class of mixed monotone operators. Z. Anal. Anwend. 22(3), 529-542 (2003)

8. $\mathrm{Wu}, \mathrm{Y}, \mathrm{Li}, \mathrm{G}$ : On the fixed point existence and uniqueness theorems of mixed monotone operators and applications. Acta Math. Sin. 46(1), 161-166 (2003) (in Chinese)

9. Li, K, Liang, J, Xiao, T: New existence and uniqueness theorems of positive fixed points for mixed monotone operators with perturbation. J. Math. Anal. Appl. 328, 753-766 (2007)

10. Lian, $X, L i$, Y: Fixed point theorems for a class of mixed monotone operators with applications. Nonlinear Anal. 67, 2752-2762 (2007)

11. $\mathrm{Wu}, \mathrm{Y}, \mathrm{Liang}, \mathrm{Z}$ : Existence and uniqueness of fixed points for mixed monotone operators with applications. Nonlinear Anal. 65, 1913-1924 (2006)

12. Wang, W, Liu, X, Cheng, S: Fixed points for mixed monotone operators and applications. Nonlinear Stud. 14(2), 189-204 (2007)

13. Zhang, Z, Wang, K: On fixed point theorems of mixed monotone operators and applications. Nonlinear Anal. 70 , 3279-3284 (2009)

14. Lakshmikantham, V, Ciric, L: Coupled fixed point theorems for nonlinear contractions in partially ordered metric spaces. Nonlinear Anal. 70, 4341-4349 (2009)

15. Zhao, Z: Existence and uniqueness of fixed points for some mixed monotone operators. Nonlinear Anal. 73 $1481-1490$ (2010)

16. Harjani, J, López, B, Sadarangani, K: Fixed point theorems for mixed monotone operators and applications to integral equations. Nonlinear Anal. 74, 1749-1760 (2011)

17. Zhai, CB, Zhang, LL: New fixed point theorems for mixed monotone operators and local existence-uniqueness of positive solutions for nonlinear boundary value problems. J. Math. Anal. Appl. 382, 594-614 (2011)

18. Zhai, CB, Hao, MR: Fixed point theorems for mixed monotone operators with perturbation and applications to fractional differential equation boundary value. Nonlinear Anal. 75, 2542-2551 (2012)

19. Lin, X, Jiang, D, Li, X: Existence and uniqueness of solutions for singular fourth-order boundary value problems. J. Comput. Appl. Math. 196, 155-161 (2006)

20. Yuan, C, Jiang, D, Zhang, Y: Existence and uniqueness of solutions for singular higher order continuous and discrete boundary value problems. Bound. Value Probl. 2008, Article ID 123823 (2008)

21. Lei, $P$, Lin, $X$, Jiang, D: Existence and uniqueness of positive solutions for singular nonlinear elliptic boundary value problems. Nonlinear Anal. 69, 2773-2779 (2008)

22. Guo, D, Lakshmikantham, V: Nonlinear Problems in Abstract Cones. Academic Press, New York (1988)

23. Zhai, CB, Guo, CM: On $\alpha$-convex operators. J. Math. Anal. Appl. 316, 556-565 (2006)

doi:10.1186/1687-1812-2013-119

Cite this article as: Zhai: Fixed point theorems for a class of mixed monotone operators with convexity. Fixed Point Theory and Applications 2013 2013:119. 